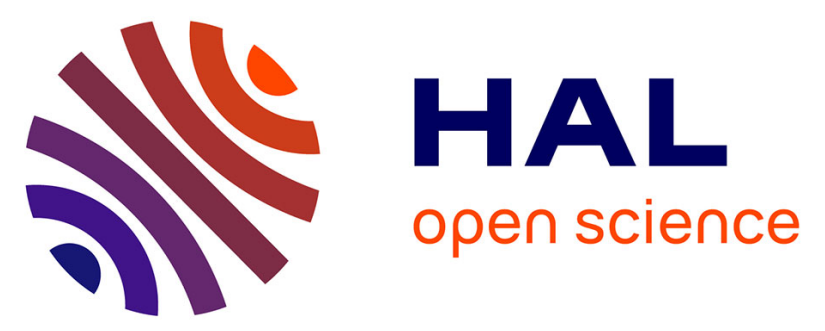

\title{
Rationale and design of the Cyclosporine to ImpRove Clinical oUtcome in ST-elevation myocardial infarction patients (the CIRCUS trial)
}

\author{
Nathan Mewton, Thien T. Cung, Olivier Morel, Guillaume Cayla, Eric
} Bonnefoy-Cudraz, Gilles Rioufol, Denis Angoulvant, Patrice Guerin, Meyer Elbaz, Nicolas Delarche, et al.

\section{To cite this version:}

Nathan Mewton, Thien T. Cung, Olivier Morel, Guillaume Cayla, Eric Bonnefoy-Cudraz, et al.. Rationale and design of the Cyclosporine to ImpRove Clinical oUtcome in ST-elevation myocardial infarction patients (the CIRCUS trial). American Heart Journal, 2015, 169 (6), pp.758-766.e6. 10.1016/j.ahj.2015.02.020 . hal-01260554

\section{HAL Id: hal-01260554}

\section{https://hal-univ-rennes1.archives-ouvertes.fr/hal-01260554}

Submitted on 3 Feb 2016

HAL is a multi-disciplinary open access archive for the deposit and dissemination of scientific research documents, whether they are published or not. The documents may come from teaching and research institutions in France or abroad, or from public or private research centers.
L'archive ouverte pluridisciplinaire HAL, est destinée au dépôt et à la diffusion de documents scientifiques de niveau recherche, publiés ou non, émanant des établissements d'enseignement et de recherche français ou étrangers, des laboratoires publics ou privés. 


\section{Rationale and Design of the Cyclosporine to ImpRove}

\section{Clinical oUtcome in ST elevation myocardial infarction}

\section{patients (the CIRCUS trial)}

\section{Authors:}

Mewton N; Cung T; Morel O; Cayla G; Bonnefoy E; Rioufol G; Angoulvant D; Guerin P; Elbaz M; Delarche N; Coste P; Vanzetto G; Metge M; Aupetit JF; Jouve B; Motreff P; Tron C; Labeque JN; Steg PG; Cottin Y; Range G; Clerc J; Coussement P; Prunier F; Moulin F; Roth O; Belle L; Dubois P; Barragan P; Gilard M; Piot C; Colin P; Morice MC; Monassier JP; Ider O; Dubois Rande P; Unterseeh T; Lebreton H; Beard T; Blanchard D; Grollier G; Malquarti V; Staat P; Sudre A; Hansson MJ; Elmer E; Boussaha I; Jossan C; Torner A; Claeys M; Garcia Dorado D; Ovize $\mathrm{M}$ and the CIRCUS Study Investigators.

RCT\# NCT01502774

\section{Corresponding author:}

Michel OVIZE, MD, PhD

Hôpital Cardiovasculaire Louis Pradel, Clinical Investigation Center of Lyon

28, Avenue Doyen Lépine, 69677 BRON Cedex, France

Tél. : +33 (4) 72357592

Fax : +33 (4) 72356910

michel.ovize@chu-lyon.fr 


\begin{abstract}

\section{Background}

Both acute myocardial ischemia and reperfusion contribute to cardiomyocyte death in STelevation myocardial infarction (STEMI). The final infarct size is the principal determinant of the subsequent clinical outcome in STEMI patients. In a proof-of-concept phase II trial, the administration of cyclosporine prior to primary percutaneous coronary intervention (PPCI) has been associated with a reduction of infarct size in STEMI patients.
\end{abstract}

\title{
Methods
}

CIRCUS is an international, prospective, multicenter, randomized, double-blinded, placebocontrolled trial. The study is designed to compare the efficacy and safety of cyclosporine versus placebo, in addition to revascularization by PPCI, in patients presenting with acute anterior myocardial infarction within 12 hours of symptoms onset and initial TIMI flow $\leq 1$ in the culprit left anterior descending coronary artery. Patients are randomized in a $1: 1$ fashion to $2.5 \mathrm{mg} / \mathrm{kg}$ intravenous infusion of cyclosporine or matching placebo performed in the minutes preceeding PCI. The primary efficacy endpoint of CIRCUS is a composite of 1-year all-cause mortality, rehospitalization for heart failure or heart failure worsening during inital hospitalization, and left ventricular adverse remodelling as determined by sequential transthoracic echochardiography. Secondary outcomes will be tested using a hierarchical sequence of left ventricular (LV) ejection fraction and absolute measurements of LV volumes. The composite of death and rehospitalization for heart failure or heart failure worsening during inital hospitalization will be further assessed at three years after the initial infarction. 


\section{Results}

Recruitment lasted from April 2011 to February 2014. The CIRCUS trial has recruited 975 patients with acute anterior myocardial infarction. The 12-months results are expected to be available in 2015.

\section{Conclusions}

The CIRCUS trial is testing the hypothesis that cyclosporine in addition to early revascularization with PPCI compared to placebo in patients with acute anterior myocardial infarction reduces the incidence of death, heart failure and adverse LV remodelling at one-year follow-up. 


\section{Background}

Studies have demonstrated that infarct size is a major determinant of prognosis after AMI ${ }^{1,2}$. Interventions aimed at reducing infarct size might therefore be of major clinical interest to improve the outcome of AMI patients. Timely reperfusion by primary percutaneous coronary intervention is the standard of care for $\mathrm{AMI}^{3}$. The progress in the access to and efficiency of primary percutaneous coronary intervention (PPCI) for ST-elevation myocardial infarction (STEMI) patients during the last decade is one of the main factors underlying the improvement in STEMI patients clinical outcomes ${ }^{4}$.

However, experimental and clinical reports indicate that reperfusion has deleterious effects, including myocardial stunning, induction of ventricular arrhythmias, no reflow and lethal reperfusion injury ${ }^{5,6}$. Irreversible damage occurring after reperfusion may account for up to $40 \%$ of the final infarct size ${ }^{7}$.

Accumulating evidence suggests that cardiomyocyte mitochondria play a key role in lethal reperfusion injury ${ }^{8-11}$. The opening of a nonspecific high conductance channel (called the mitochondrial permeability transition pore) in the inner mitochondrial membrane results in the collapse of the membrane potential, the uncoupling of the respiratory chain, the release of cytochrome $\mathrm{c}$ and other proapoptotic factors and the depletion of ATP; these metabolic alterations may lead to cardiomyocyte death ${ }^{12}$. Calcium overload and excessive production of reactive oxygen species caused by sudden reperfusion of the ischemic myocardium can trigger the opening of the mitochondrial permeability transition pore ${ }^{9,12-14}$.

In addition to its well-known immunosuppressive properties, cyclosporine is a potent inhibitor of mitochondrial permeability transition pore ${ }^{15}$. Several reports indicate that cyclosporine given at the time of reperfusion limits lethal reperfusion injury under experimental conditions $9,16-18$. 
Recently, a small proof-of-concept clinical study in STEMI patients showed that cyclosporine given immediately prior to reperfusion could reduce infarct size as measured by CMR by approximately $20 \%{ }^{19}$.

This underlines the need for a phase III randomized trial to address the clinical efficacy of cyclosporine in addition to PPCI for acute myocardial infarction patients. The CIRCUS trial was designed to test the hypothesis that a single intravenous administration of cyclosporine immediately before PCI reduces the 1-year composite of mortality, rehospitalization for heart failure or heart failure worsening during initial hospitalization, and left ventricular remodelling in anterior STEMI patients referred for PPCI. 


\section{Study objectives}

In the CIRCUS trial, patients with anterior STEMI undergoing PPCI are randomized to a single intravenous bolus of $2.5 \mathrm{mg} / \mathrm{kg}$ of cyclosporine (Ciclomulsion ${ }^{\circledR}$, NeuroVive Pharmaceutical AB, Lund, Sweden) or $2.5 \mathrm{mg} / \mathrm{kg}$ of matching placebo.

The primary objective of the study is to determine whether the administration of a single intravenous bolus of cyclosporine before reperfusion compared to placebo in patients with large amounts of myocardium at risk (anterior STEMI) results in reduced incidence of all-cause mortality, rehospitalization for heart failure or heart failure worsening during initial hospitalization and adverse left ventricular (LV) remodelling as assessed by transthoracic echocardiography at 1-year follow-up. Adverse LV remodelling is defined here as an increase in LV end-diastolic volume (LVEDV) $\geq 15 \%$ between the initial LVEDV and follow-up LVEDV at one-year.

The reasons for choosing this primary objective are:

- all-cause mortality is a hard clinical endpoint and in the first-year following anterior MI, the main cause of death is of cardio-vascular origin. In addition, in this off-label use of cyclosporine, it appeared mandatory to have all-cause death as an endpoint. This is to account for any potential unexpected cardiac or extra-cardiac effects of cyclosporine.

- heart failure events are closely related to the amount of myocardial tissue damage induced by the ischemic and reperfusion injuries ${ }^{2}$. As our primary hypothesis is that cyclosporine significantly reduces infarct size, we expect a significant reduction of heart failure events after AMI.

- adverse LV remodelling is closely related to the final infarct size ${ }^{20}$. If cyclosporine significantly reduces the final infarct size, it should also reduce the adverse LV remodelling 
process. The link between the adverse remodelling process and hard clinical events is unclear. The long-term follow-up at 3-years of the CIRCUS study should provide evidence for this relationship.

An important secondary objective is to evaluate the clinical outcome at 36 months defined by the composite endpoint of death and rehospitalization for heart failure or heart failure worsening during inital hospitalization.

\section{Study population and patient selection}

The CIRCUS study is a prospective, randomized, international, multicenter, placebo-controlled trial in patients with anterior acute STEMI referred for PPCI.

The study is ongoing in 57 centers in France, Belgium and Spain. The study is being performed according to the principles of the Declaration of Helsinki (1964, and its text revisions), and approval has been obtained from ethics committees in the countries concerned. The trial has been registered under www.clinicaltrials.gov: NCT01502774 and EudraCT number: 2009-013713-99.

The study population consists of 975 anterior acute STEMI patients enrolled at 52 centers in France, 4 centers in Belgium and 1 in Spain.

Recruitment started April 2011 and was completed in February 2014. The final results of the oneyear clinical composite endpoint are expected end of Q2 2015.

The main inclusion and exclusion criteria are listed in Table 1.

Consent was obtained in an emergency setting. In all centers, patients were informed as soon as possible of the ongoing trial prior to coronary angiography. The angiogram was performed to collect TIMI flow data. If the selection criteria were completed, then the informed consent was 
obtained. If the written informed consent could not be obtained, an oral informed consent was obtained and ascertained by an impartial witness, independent of study investigator, or a patient's legal representative or relative. In the latter case, the study investigator obtained the patient's written informed consent as soon as possible after randomization.

\section{Randomization and treatment protocol}

The investigation schedule is depicted in Figure 1 and the study flow chart is shown in Table 2. Patient randomization was performed centrally by internet after secure login into the on-line casereport form (CRF) of the study, with a randomization ratio of 1:1 using a computer-based randomization program, stratified by center.

All patients received revascularization by PPCI plus medical treatment according to guidelines ${ }^{3}$. During PCI, the choice of stent (bare-metal or drug-eluting stent) was left to the discretion of the operator. The use of thrombus aspiration, intra-coronary glycoprotein IIb/IIIa inhibition and devices to prevent distal embolization was left to the discretion of the operator.

After coronary angiography but before angioplasty, patients who met the enrolment criteria were randomly allocated to either the placebo or the cyclosporine group. Before the first balloon inflation, patients received an intravenous bolus injection of $2.5 \mathrm{mg} / \mathrm{kg}$ of the study drug. For each group, the study drug was injected slowly (over 2 to 3 minutes) via a catheter positioned within a peripheral vein.

The investigational medicinal product is cyclosporine (Ciclomulsion ${ }^{\circledR}, \quad$ NeuroVive Pharmaceutical AB, Lund, Sweden) ${ }^{21}$. The matching placebo of Ciclomulsion ${ }^{\circledR}$ (NeuroVive Pharmaceutical AB, Lund, Sweden) is a lipid emulsion composed with refined soya-bean oil, medium-chain triglycerides, egg lecithin, water-free glycerol, sodium oleate, sodium hydroxide, 
water for injection). The qualitative composition of Ciclomulsion ${ }^{\circledR}$ and its placebo only differed by the presence or absence of cyclosporine. The final emulsions were visually indistinguishable. All study procedures are detailed in Table 2. 12-lead electrocardiogram, coronary angiography and echocardiographic data procedures are presented below.

Assessment of the clinical outcomes will be performed by 3-telephone interviews at 30-day, 3month and 6 month follow-up. The primary outcome will be measured during a routine cardiology visit performed at 1 - and 3-years.

\section{Electrocardiogram (ECG)}

For all patients five 12-lead ECGs will be obtained at admission (before PCI), after PCI (60 to 90 minutes after PCI), at hospital discharge, at 1 year ( \pm 8 weeks) and 3 years ( \pm 8 weeks). All ECG recordings will be sent to the coordination center for a centralised reading in the CIRCUS ECGcore laboratory. Expert readers will assess the maximal ST-segment deviation in the anterior leads and the number of significant $Q$ waves for each recording.

\section{Coronary Angiography}

For all patients, the coronary angiography performed at admission will be stored digitally and sent to the coordinating center for centralized reading. All angiographic data will be analyzed on a specific workstation (Osirix) by a single expert observer blinded to all other clinical data. Site of occlusion, initial and final TIMI flow, collateral circulation (Rentrop grade), stent number, multi-vessel disease status, coronary interventions and complications, angiographic estimate of the area at risk size (APPROACH score ${ }^{22}$ ) will be assessed for each patient. 


\section{Cardiac biomarkers}

For all patients, at least two blood samples for cardiac biomarkers (total creatine kinase (CK), troponin $\mathrm{T}$ or $\mathrm{I})$ will be performed and measured locally. Biomarkers will be assessed at admission and at 4 hours ( \pm 30 minutes) after reperfusion.

\section{Transthoracic Echocardiography}

For all patients, conventional transthoracic echocardiography will be performed in all patients after PCI during the index hospitalization (within the first week following admission), at one year ( \pm 8 weeks) and 3 years $( \pm 8$ weeks) after AMI.

Patients are scanned in the left supine position from an apical window. Images are obtained using a $3.5 \mathrm{MHz}$ transducer, at a depth of 12 to $20 \mathrm{~cm}$ in parasternal and apical views. Three cardiac cycles of the apical 4-, 3-, and 2-chamber views are captured in 2D.

Left ventricular volumes are measured and the biplane Simpson's rule is applied ${ }^{23,24}$. LV remodelling will be assessed at 1 year ( \pm 8 weeks) versus baseline echocardiography (48 hours \pm 48 hours) according to the following formula:

(LVEDV 1 year-LVEDV baseline/ LVEDV baseline) x 100.

All echocardiographic data are stored digitally and sent to the coordinating center for a centralized corelab reading. Two blinded expert operators will centrally assess data on a specific workstation.

\section{Primary and secondary end points}

The primary end point is a composite of 1-year all-cause mortality, rehospitalization for heart failure or heart failure worsening during inital hospitalization, and left ventricular adverse remodelling as determined by sequential transthoracic echochardiography. 
Key secondary endpoints include LV ejection fraction (LVEF), LV end-diastolic and end-systolic volumes assessed within the first week following admission and at one and three years.

Secondary endpoints also include all individual components of the primary endpoint taken separately (all cause mortality; rehospitalization for heart failure or worsening of initial heart failure status during initial hospitalization; adverse LV remodelling).

Other secondary endpoints used in several previous STEMI trials ${ }^{25,26}$ are being examined across both arms. They include the following:

1) Major adverse ischemic cardiac events: unstable angina or reinfarction (using the universal definition of myocardial infarction definition ${ }^{27}$ ) and unplanned revascularization by either PCI or coronary artery bypass graft;

2) Major adverse cardiac and cerebrovascular events;

3) Implantable cardioverter defibrillator implantation for primary or secondary prevention;

4) NYHA (New York Heart Association) dyspnea status at one and three years;

5) Incidence of complete ST-segment resolution $60 \pm 30$ minutes after the last angiogram;

6) 4 \pm 2 -hours post-admission levels of serum troponin and creatine kinase;

We did not have the means to assess the myocardial biomarker release kinetics after reperfusion conversely to our previous trial ${ }^{19}$. The sample size, the change in local routine practices and the heterogeneity of biomarker assays (especially for hypersensitive troponins) prevented us from comparing the biomarker release between both groups. Post-reperfusion LV arrhythmias (nonsustained and sustained ventricular tachycardia and ventricular fibrillation) will also be compared between both groups, as another exploratory endpoint.

Besides efficacy, the trial addresses the issue of safety of a $2.5 \mathrm{mg} / \mathrm{kg}$ cyclosporine intravenous bolus given immediately prior to reperfusion. Safety assessment includes acute renal failure as 
assessed by serial measurement of creatinine between baseline and 48 hours after admission and any other serious adverse events declared by investigational centers as being potentially related to investigative drug/placebo.

\section{Substudy}

The CIRCUS trial has one Cardiac Magnetic Resonance Imaging sub-population analysis. It plans to evaluate infarct size, microvascular obstruction, LV remodelling and LV systolic function recovery. This exploratory sub-population analysis will be performed only in centers fulfilling technical requirements and where CMR is available in routine practice ${ }^{28,29}$. There is no prespecified sample size for this sub-population analysis.

\section{Study monitoring and adjudication committee}

An independent Data Safety Monitoring Board (DSMB) that includes one cardiologist, one pharmacologist and one bio-statistician will monitor the trial. The DSMB will receive the results of two blinded interim analyses performed after 300 and 500 inclusions. Interim safety analyses will assess the one-month all-cause mortality and acute renal failure in both study groups. The DSMB will be entitled to make recommendations to the steering committee regarding the continuation of the study.

All clinical and safety endpoints will be adjudicated by a Clinical Endpoints Committee (CEC), blinded to the patients' assigned treatment, based on data provided by the investigational centers. 


\section{Study Funding}

The CIRCUS trial is an investigator-initiated trial that is supported by a national PHRC program funded by the French Ministry of Health (PHRC National 2010). Furthermore, it is supported by grants from NeuroVive Pharmaceutical AB, Lund, Sweden, that also provides the study treatment Ciclomulsion ${ }^{\circledR}$ and matching placebo.

\section{Statistical design}

We estimated that the proportion of patients reaching the endpoints (death, hospitalization for heart failure (HF), LV remodelling) in the control group over the planned 12 months of the study would be $7 \%$ for death and $42 \%$ for heart failure and LV remodelling combined. The expected proportion of the combined endpoint was estimated to $49 \%$ taking into account the overlap of heart failure and LV remodelling. An estimated 790 patients (395 patients per group) would be required for the study to have $80 \%$ power to detect a $20 \%$ reduction in the relative risk with a two-sided alpha level of 5\%. We estimated that the assessment of LV remodelling would be missing for $14 \%$ patients surviving at 1 year, and the sample size was increased to adjust for missing values. In addition, a further $10 \%$ was added to accommodate the fact that the sample size was calculated assuming the use of a chi-square test while a different model will be used for the analysis. The final sample size was estimated to 972 patients. The sample size was calculated using nQuery+nTerim 2.0.

The primary analysis will be a modified intention-to-treat (mITT) analysis including all randomized patients with a valid measurement for the primary endpoint. Patients will be analysed in the treatment group to which they were allocated by randomization. 
For the primary endpoint, data will be analysed by comparing the proportion of the primary combined efficacy endpoint at 12 months in the active group vs. the placebo group by using a logistic mixed effect regression model that includes treatment as fixed effect and center as random effect.

A hierarchial approach will be used to adjust for multiple testing of the first five endpoints:

a) Combined incidence of (death, heart failure and increase in LVEDV $>15 \%$ at one year),

b) Ejection fraction (EF \%) at 12 months

c) Left Ventricular End Diastolic Volume (LVEDV) at 12 months

d) Left Ventricular End Systolic Volume (LVESV) at 12 months

e) Time to first event (death, hospitalization for heart failure) at 36 months.

The abovementioned endpoint e), time to first event (death, hospitalization for heart failure) is classified as an additional primary endpoint at 36 months but will follow as endpoint number five in the hierarchial testing. This since the other endpoints in the hierarchy will be analysed and reported at 12 months. Each subsequent test will only be considered as confirmatory providing the previous step(s) is/are successful at the $5 \%$-level $(\mathrm{p}<0.05)$. If any of the previous steps is not successful, the analysis of the following steps will be considered descriptive.

Treatment difference in EF at 12 months, LVEDV at 12 months and LVESV at 12 months will be estimated and analysed using mixed linear models that include treatment as fixed effect and centre as random effect. Other covariates may be added for additional analyses of the secondary endpoints. Where applicable, non-parametric methods may be considered as alternative approach for some of the endpoints. 
For each component of the primary endpoint and for secondary clinical outcomes endpoints such cardiovascular mortality, myocardial infarction, unstable angina, stroke, descriptive methods without formal statistical testing will be used.

The primary analysis of CIRCUS is conducted at 12 months and at 36 months a follow-up analysis will be performed. The purpose of the 36-month analysis is to gain additional power for an additional combined endpoint of hard outcomes: time to first event [all-cause mortality, hospitalization for heart failure] at three years post-AMI.

An exploratory analysis is to be conducted at 36 months to evaluate the predictive value of Left Ventricular End Diastolic Volume at 12 months for Major Cardiac Adverse Events (MACE) occurring during the subsequent 2 years follow-up. This analysis will use epidemiological statistical methods such as ROC (Receiver Operating Characteristics) regression and Cox regression, possibly also including a classical Prentice analysis ${ }^{32}$.

\section{Study organization}

The CIRCUS Steering Committee is chaired by Michel Ovize, MD, PhD of the Claude Bernard University of Lyon1 - Hôpital Cardiovasculaire Louis Pradel, Lyon, France. Members of the committees and corelabs and participating centers with all principal investigators and subinvestigators are listed in the online Appendix A. The Steering Committee is responsible for the scientific content of the protocol and oversees the trial operations and will prepare the primary manuscript and other publications arising from the CIRCUS Trial.

Each participating center has a senior investigator with clinical trial experience. All trial-related processes will follow the standard operating procedures of the CIRCUS study protocol. The Clinical Investigation Center (CIC) located in Lyon, France, is the coordinating center of the 


\section{CIRCUS study.}

Monitoring on site of all centers is performed by the Study Sponsor, the Hospices Civils de Lyon (Clinical Research and Innovation Direction).

The authors are solely responsible for the design and conduct of this study, all study analyses, the drafting and editing of the manuscript, and its final contents.

\section{Discussion}

CIRCUS is the largest prospective phase III randomized trial assessing the impact of a single injection of cyclosporine compared to placebo just before revascularization on a composite endpoint of all-cause mortality, rehospitalization for heart failure, and echocardiographic LV remodelling at 1-year follow up in acute STEMI patients referred to PPCI.

During the past two decades, several negative studies have contributed to the growing skepticism regarding the ability of any intervention to reduce infarct size in acute MI patients. From the early 2000s on, this pessimistic view of infarct size reduction has been reconsidered. First, major improvements in reperfusion therapy have helped interventional cardiologists to better control the conditions of myocardial reperfusion in the settings of acute coronary syndromes. Second, the description of the phenomenon of ischemic postconditioning in the dog model in 2003 was confirmed in STEMI patients in $2005^{33,34}$. This contributed to confirm the existence of lethal reperfusion injury in STEMI patients and eventually to consider its treatment ${ }^{35}$.

As a matter of fact, several small-size proof-of-concept studies have demonstrated that ischemic conditioning interventions, administered before, or immediately at the onset of reflow, were able to reduce infarct size, attenuate myocardial no-reflow and edema and improve functional recovery ${ }^{33,36,37}$. Because ischemic postconditioning by coronary angioplasty is not always easy 
to handle (thrombotic burden, complex tortuous coronary anatomy), some investigators examined whether pharmacological agents might mimic ischemic postconditioning.

Piot et al. were the first to demonstrate that a single bolus intravenous administration of cyclosporine prior to PCI reperfusion could reduce infarct size in STEMI patients ${ }^{19,38}$. However, another trial failed to find benefit of cyclosporine in combination to thrombolysis ${ }^{39}$. In the MitoCare trial, administration of TRO40303, an agent that binds the outer mitochondrial membrane but does not act directly on the mitochondrial permeability transition pore, failed to reduce infarct size in STEMI patients ${ }^{40}$.

In a follow-up study of the Piot's trial, Mewton et al. reported that patients who received cyclosporine immediately prior to PCI developed less adverse LV remodelling and displayed improved LV function at 6 months post-infarction ${ }^{38}$. In addition, recent phase II trials have demonstrated that other pharmacological agents, administered prior to reperfusion, may reduce infarct size in STEMI patients, including intra-coronary abciximab, metoporolol or the GLP-1 analog exenatide ${ }^{26,41,42}$.

However, these encouraging results remain of limited clinical relevance. A pragmatic and sufficiently powered trial assessing a cardioprotective intervention on well-defined clinical endpoints is needed for the confirmation of a significant clinical benefit and future implementation on a routine basis. The CIRCUS trial was thus designed with the primary objective to examine the potential benefit of cyclosporine on outcomes after STEMI.

Beyond the evaluation of the impact of cyclosporine on clinical endpoints, the results are also expected to shed further light on the role of LV remodelling as a determinant of post-AMI heart failure $^{20,30}$. At 36 months, a composite endpoint of death and rehospitalization for heart failure or worsening of heart failure during initial hospitalization will be analysed as a key secondary endpoint. The longer follow-up will yield power for this more conventional endpoint based 
exclusively on hard outcomes.

The study population was defined in order to maximize the ability to detect a benefit of cyclosporine in preventing adverse clinical outcomes related to lethal reperfusion injury. Cyclosporine, as well as ischemic conditioning interventions, is known to be of potentially greater benefit in patients with a large area at risk $^{16,19,38}$. Patients included in the CIRCUS trial display large areas at risk (anterior STEMI), and have an increased risk of adverse LV remodelling and worse clinical outcomes ${ }^{2,30}$. The CIRCUS trial was designed in a pragmatic way, i.e. the study treatment was administered as an adjunct to standard care, easy to add to the routine clinical practice in the catheterization laboratory. If this trial were positive, with a significant effect on all three components of the primary composite endpoint, there would be a rationale to recommend intravenous cyclosporine administration prior to reperfusion in patients presenting with anterior MI and an occluded LAD artery. Provided no significant side effects induced by cyclosporine were shown. The extension to all-comer STEMI patients with various initial TIMI status and in different infarct territories at inclusion would need further trials in these specific clinical situations.

\section{Summary}

Proof-of-concept phase II trials have tested the use of protective strategies in AMI to reduce the reperfusion-induced damage to the myocardium. The CIRCUS trial has been designed to test the hypothesis that on a background of early revascularization with PPCI, cyclosporine in comparison with placebo will improve the one-year and three-year outcomes of patients with acute anterior myocardial infarction. 


\section{References}

1. Gibbons RJ, Valeti US, Araoz PA et al. The quantification of infarct size. J Am Coll Cardiol 2004;44(8):1533-1542.

2. Lonborg J, Vejlstrup N, Kelbaek H et al. Final infarct size measured by cardiovascular magnetic resonance in patients with ST elevation myocardial infarction predicts long-term clinical outcome: an observational study. Eur Heart J Cardiovasc Imaging 2013;14(4):387395.

3. Steg PG, James SK, Atar D et al. ESC Guidelines for the management of acute myocardial infarction in patients presenting with ST-segment elevation: The Task Force on the management of ST-segment elevation acute myocardial infarction of the European Society of Cardiology (ESC). Eur Heart J 2012;33(20):2569-2619.

4. Puymirat E, Simon T, Steg PG et al. Association of changes in clinical characteristics and management with improvement in survival among patients with ST-elevation myocardial infarction. JAMA 2012;308(10):998-1006.

5. Reffelmann T, Kloner RA. The no-reflow phenomenon: A basic mechanism of myocardial ischemia and reperfusion. Basic Res Cardiol 2006;101(5):359-372.

6. Rezkalla SH, Kloner RA. No-reflow phenomenon. Circulation 2002;105(5):656-662.

7. Yellon DM, Hausenloy DJ. Myocardial reperfusion injury. N Engl J Med 2007;357(11):1121-1135.

8. Baines CP, Kaiser RA, Purcell NH et al. Loss of cyclophilin D reveals a critical role for mitochondrial permeability transition in cell death. Nature 2005;434(7033):658-662.

9. Di Lisa F, Menabo R, Canton M et al. Opening of the mitochondrial permeability transition 
pore causes depletion of mitochondrial and cytosolic NAD + and is a causative event in the death of myocytes in postischemic reperfusion of the heart. J Biol Chem 2001;276(4):25712575.

10. Griffiths EJ, Halestrap AP. Mitochondrial non-specific pores remain closed during cardiac ischaemia, but open upon reperfusion. Biochem J 1995;307(Pt 1):93-98.

11. Leung AW, Halestrap AP. Recent progress in elucidating the molecular mechanism of the mitochondrial permeability transition pore. Biochim Biophys Acta 2008;1777(7-8):946952.

12. Javadov S, Karmazyn M. Mitochondrial permeability transition pore opening as an endpoint to initiate cell death and as a putative target for cardioprotection. Cell Physiol Biochem 2007;20(1-4):1-22.

13. Crompton M. The mitochondrial permeability transition pore and its role in cell death. Biochem J 1999;341(Pt 2):233-249.

14. Morota S, Manolopoulos T, Eyjolfsson A et al. Functional and pharmacological characteristics of permeability transition in isolated human heart mitochondria. PLoS One 2013;8(6):e67747.

15. Duchen MR, McGuinness O, Brown LA et al. On the involvement of a cyclosporin A sensitive mitochondrial pore in myocardial reperfusion injury. Cardiovasc Res 1993;27(10):1790-1794.

16. Argaud L, Gomez L, Gateau-Roesch $\mathrm{O}$ et al. Trimetazidine inhibits mitochondrial permeability transition pore opening and prevents lethal ischemia-reperfusion injury. J Mol Cell Cardiol 2005;39(6):893-899.

17. Skyschally A, Schulz R, Heusch G. Cyclosporine A at reperfusion reduces infarct size in pigs. Cardiovasc Drugs Ther 2010;24(1):85-87. 
18. Chiari P, Angoulvant D, Mewton N et al. Cyclosporine protects the heart during aortic valve surgery. Anesthesiology 2014;121(2):232-238.

19. Piot C, Croisille $\mathrm{P}$, Staat $\mathrm{P}$ et al. Effect of cyclosporine on reperfusion injury in acute myocardial infarction. N Engl J Med 2008;359(5):473-481.

20. Savoye C, Equine O, Tricot $\mathrm{O}$ et al. Left ventricular remodelling after anterior wall acute myocardial infarction in modern clinical practice (from the REmodelage VEntriculaire [REVE] study group). Am J Cardiol 2006;98(9):1144-1149.

21. Ehinger KH, Hansson MJ, Sjovall $\mathrm{F}$ et al. Bioequivalence and tolerability assessment of a novel intravenous ciclosporin lipid emulsion compared to branded ciclosporin in Cremophor (R) EL. Clin Drug Investig 2013;33(1):25-34.

22. Ortiz-Perez JT, Meyers SN, Lee DC et al. Angiographic estimates of myocardium at risk during acute myocardial infarction: validation study using cardiac magnetic resonance imaging. Eur Heart J 2007;28(14):1750-1758.

23. Douglas PS, Garcia MJ, Haines et al. ACCF/ASE/AHA/ASNC/HFSA/HRS/SCAI/SCCM/SCCT/SCMR 2011 Appropriate Use Criteria for Echocardiography. A Report of the American College of Cardiology Foundation Appropriate Use Criteria Task Force, American Society of Echocardiography, American Heart Association, American Society of Nuclear Cardiology, Heart Failure Society of America, Heart Rhythm Society, Society for Cardiovascular Angiography and Interventions, Society of Critical Care Medicine, Society of Cardiovascular Computed Tomography, Society for Cardiovascular Magnetic Resonance American College of Chest Physicians. J Am Soc Echocardiogr 2011;24(3):229-267.

24. Picard MH, Adams D, Bierig SM et al. American Society of Echocardiography recommendations for quality echocardiography laboratory operations. J Am Soc 
Echocardiogr 2011;24(1):1-10.

25. Lonborg J, Holmvang L, Kelbaek H et al. ST-Segment resolution and clinical outcome with ischemic postconditioning and comparison to magnetic resonance. Am Heart $\mathrm{J}$ 2010;160(6):1085-1091.

26. Stone GW, Maehara A, Witzenbichler B et al. Intracoronary abciximab and aspiration thrombectomy in patients with large anterior myocardial infarction: the INFUSE-AMI randomized trial. JAMA 2012;307(17):1817-1826.

27. Thygesen K, Alpert JS, Jaffe AS et al. Third universal definition of myocardial infarction. Eur Heart J 2012;33(20):2551-2567.

28. Mewton N, Thibault H, Roubille F et al. Postconditioning attenuates no-reflow in STEMI patients. Basic Res Cardiol 2013;108(6):383.

29. Thuny F, Lairez O, Roubille F et al. Post-conditioning reduces infarct size and edema in patients with ST-segment elevation myocardial infarction. J Am Coll Cardiol 2012;59(24):2175-2181.

30. Bolognese L, Neskovic AN, Parodi G et al. Left ventricular remodelling after primary coronary angioplasty: patterns of left ventricular dilation and long-term prognostic implications. Circulation 2002;106(18):2351-2357.

31. Ferrari R. Effects of angiotensin-converting enzyme inhibition with perindopril on left ventricular remodelling and clinical outcome: results of the randomized Perindopril and Remodelling in Elderly with Acute Myocardial Infarction (PREAMI) Study. Arch Intern Med 2006;166(6):659-666.

32. Prentice RL. Surrogate endpoints in clinical trials: definition and operational criteria. Stat Med 1989;8(4):431-440.

33. Staat P, Rioufol G, Piot $\mathrm{C}$ et al. Postconditioning the human heart. Circulation 
2005;112(14):2143-2148.

34. Zhao ZQ, Corvera JS, Halkos ME et al. Inhibition of myocardial injury by ischemic postconditioning during reperfusion: comparison with ischemic preconditioning. Am J Physiol Heart Circ Physiol 2003;285(2):H579-H588.

35. Ovize M, Baxter GF, Di Lisa F et al. Postconditioning and protection from reperfusion injury: where do we stand? Position paper from the Working Group of Cellular Biology of the Heart of the European Society of Cardiology. Cardiovasc Res 2010;87(3):406-423.

36. Lonborg J, Kelbaek H, Vejlstrup $\mathrm{N}$ et al. Cardioprotective effects of ischemic postconditioning in patients treated with primary percutaneous coronary intervention, evaluated by magnetic resonance. Circ Cardiovasc Interv 2010;3(1):34-41.

37. Thibault H, Piot C, Staat $\mathrm{P}$ et al. Long-term benefit of postconditioning. Circulation 2008;117(8):1037-1044.

38. Mewton N, Croisille P, Gahide G et al. Effect of cyclosporine on left ventricular remodelling after reperfused myocardial infarction. J Am Coll Cardiol 2010;55(12):12001205.

39. Ghaffari S, Kazemi B, Toluey $\mathrm{M}$ et al. The effect of prethrombolytic cyclosporine-A injection on clinical outcome of acute anterior ST-elevation myocardial infarction. Cardiovasc Ther 2013;31(4):e34-e39.

40. Atar D, Arheden H, Berdeaux A et al. Effect of intravenous TRO40303 as an adjunct to primary percutaneous coronary intervention for acute ST-elevation myocardial infarction: MITOCARE study results. Eur Heart J 2015;36(2):112-119.

41. Ibanez B, Macaya C, Sanchez-Brunete V et al. Effect of early metoprolol on infarct size in ST-segment-elevation myocardial infarction patients undergoing primary percutaneous coronary intervention: the Effect of Metoprolol in Cardioprotection During an Acute 
Myocardial Infarction (METOCARD-CNIC) trial. Circulation 2013;128(14):1495-1503.

42. Lonborg J, Vejlstrup N, Kelbaek H et al. Exenatide reduces reperfusion injury in patients with ST-segment elevation myocardial infarction. Eur Heart J 2012;33(12):1491-1499.

\section{Figure Legends}

Figure 1: The Circus Trial Timeline. LAD: left anterior descending coronary artery; LVEDV: left ventricular end-diastolic volume; PCI: percutaneous coronary intervention; STEMI: STelevation myocardial infarction; TTE: transthoracic echocardiography; TIMI: thrombolysis in myocardial infarction. 
Table 1: Main inclusion and exclusion criteria in the CIRCUS study

\section{Inclusion criteria}

1) Male and female aged $\geq 18 \mathrm{y}$, without any legal protection measure

2) Proper health insurance coverage

3) Presentation within 12 hours of symptom onset (chest pain, chest discomfort)

4) ST segment elevation $\geq 0.2 \mathrm{mV}$ in two contiguous anterior (V1-V6) leads on regular 12-lead electrocardiogram

5) Referral to cardiac catheterization laboratory for primary percutaneous coronary intervention (PPCI) or rescue PCI

6) Culprit coronary artery: Left Anterior Descending (LAD) coronary artery

7) Initial angiographic Thrombolysis in Myocardial Infarction (TIMI) flow grade $\leq 1$ in LAD

8) Informed consent obtention

\section{Exclusion criteria}

1) Unconsciousness or confusion

2) Cardiogenic shock

3) Culprit coronary artery other than LAD (left circumflex or right coronary artery), and evidence of coronary collaterals to the myocardial risk region (Rentrop grade $\geq 1$ )

4) Initial angiographic TIMI flow grade $\geq 2$ in LAD

5) History of hypersensitivity to cyclosporine

6) History of hypersensitivity to egg, peanut or Soya-bean proteins

7) History of chronic renal insufficiency (either creatinin clearance $<30 \mathrm{ml} / \mathrm{min} / 1.73 \mathrm{~m}^{2}$ or current medical care for severe renal insufficiency)

8) History of liver insufficiency 
9) Uncontrolled hypertension at the time of admission (Systolic blood pressure $>180 \mathrm{mmHg}$ and/or diastolic blood pressure $>110 \mathrm{mmHg}$ )

10) Current medication with any compound containing Hypericum perforatum (St.-John's-worth) or Stiripentol or Aliskiren or Bosentan or Rosuvastatine

11) Female patients currently pregnant or women of childbearing age without efficient contraception

12) Any disorder associated with immunological dysfunction $\leq 6$ months prior to presentation (cancer, lymphoma or known positive serology for HIV, or hepatitis)

13) Participation in another clinical trial 
Table 2 : Diagram of data acquisition

\begin{tabular}{|c|c|c|c|c|c|c|}
\hline & Hospitalization & $\begin{array}{l}1 \text { month } \\
( \pm 2 w k)\end{array}$ & $\begin{array}{l}3 \text { months } \\
( \pm 2 w k) \\
\end{array}$ & $\begin{array}{l}6 \text { months } \\
\underline{( \pm 2 w k)}\end{array}$ & $\begin{array}{l}1 \text { year } \\
\underline{( \pm 8 w k)} \\
\end{array}$ & $\begin{array}{l}3 \text { years } \\
( \pm 8 w k) \\
\end{array}$ \\
\hline & & Phone-call & Phone-call & Phone-call & $\begin{array}{l}\text { Clinical } \\
\text { visit }\end{array}$ & $\begin{array}{l}\text { Clinical } \\
\text { visit }\end{array}$ \\
\hline Written informed consent & $\mathbf{X}$ & & & & & \\
\hline Coronary-angiography & $\mathbf{X}$ & & & & & \\
\hline Study treatment administration & $\mathbf{X}$ & & & & & \\
\hline Coronary angioplasty & $\mathbf{X}$ & $y$ & & & & \\
\hline Clinical examination & $\mathbf{X}^{0}$ & & & & $\mathbf{X}$ & $\mathbf{X}$ \\
\hline $\begin{array}{l}\text { ECG at admission (before and after } \\
\text { PCI) }\end{array}$ & & & & & $\mathbf{X}$ & $\mathbf{X}$ \\
\hline $\begin{array}{l}\text { Cardiac biomarkers (total creatine } \\
\text { kinase, troponin } \mathrm{T} \text { or I) }\end{array}$ & $X^{1}$ & & & & & \\
\hline Serum creatinine, Sodium, Potassium & $X^{2}$ & & & & & \\
\hline $\begin{array}{l}\text { Glycemia, Total Bilirubin, Alkaline } \\
\text { phosphatases, ALT, AST, } \gamma \text {-GT, } \\
\text { white-cell count }\end{array}$ & $X^{2}$ & & & & & \\
\hline Transthoracic Echocardiography & $\mathbf{X}$ & & & & $\mathbf{X}$ & $\mathbf{X}$ \\
\hline Blood pressure & $\mathrm{X}^{3}$ & & & & $\mathbf{X}$ & $\mathbf{X}$ \\
\hline Heart rate & $\mathrm{X}^{3}$ & & & & $\mathbf{X}$ & $\mathbf{X}$ \\
\hline NT-Pro BNP or BNP & $\mathrm{X}^{3}$ & & & & $\mathbf{X}$ & $\mathbf{X}$ \\
\hline Quality of life questionnaire & & & & & & $\mathbf{X}$ \\
\hline
\end{tabular}




\begin{tabular}{|l|c|c|c|c|c|c|}
\hline $\begin{array}{l}\text { AE and SAE records during clinical } \\
\text { visits }\end{array}$ & $\mathbf{X}$ & & & & $\mathbf{X}$ & $\mathbf{X}$ \\
\hline SAE records during phone calls & & $\mathbf{X}$ & $\mathbf{X}$ & $\mathbf{X}$ & & \\
\hline
\end{tabular}



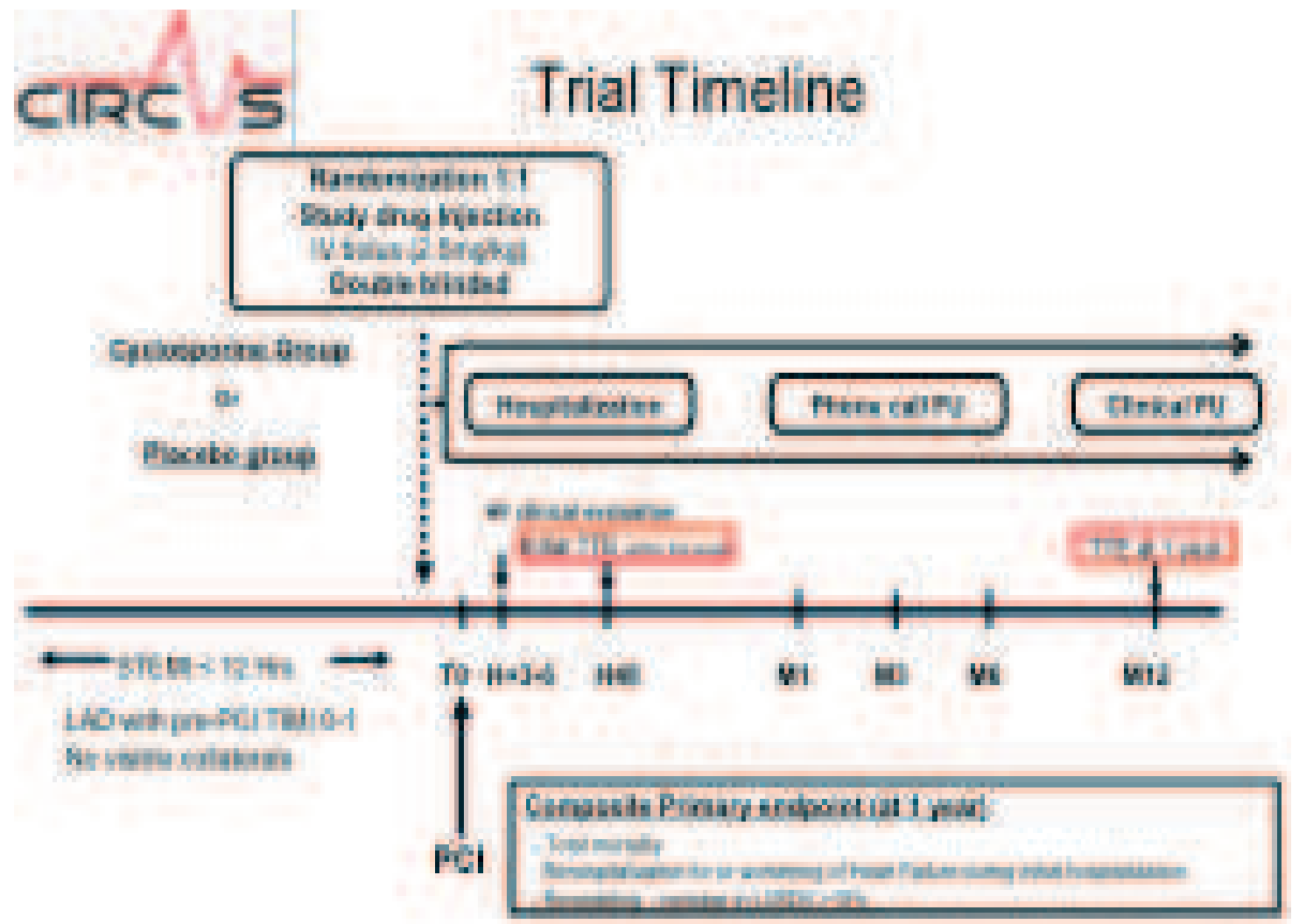

Figure 1 